\title{
Redaktionsstab des Chemischen Zentralblattes
}

Chefredakteure: Dr. phil. Eugen Klever und Dr. phil. Willi Barz

Redakteure für:

Teil A: Dr. Eugen Klever, Dr. Armand Blaschette, Dipl.Ing. Ruth Mattner, Dipl.-Phys. Herbert Weber, Dr.-Ing. (hristian Weiske. Wissenschaftliche Redaktionsmitglieder: Prof. Dr.-Ing. Bertold Reuter, Dr. Gerhard Schmidt.

Teil B: Dr. Eugen Herr, Dr.-Ing. Günter Poetzscher, Dr.-Ing. Wolfgang Schramm. Wissenschaftliches Redaktionsmitglied: Prof. Dr.-Ing. Günther Kresze.

Teil C: Dr. Eugen Herr, Dipl.-Chem. Peter Golinske, Dr. rer. nat. Margaretha Mengelberg, Dipl.-Ing. Gerhard Reisner, Dr.-Ing. Ilse Spaeth.

Teil D: Dr. Eugen Herr.

Teil E: Dr. Paul Loch, Dr. Willi Barz, Dr. Else Brandt, Dr. Irmtraut Hahn, Dipl.-Ing. Heinz Kockert, Dipl.-Biol. Margot Metze.

Teil F: Dr. Will Barz, Dipl.-Ing. Frigga Thomas.

Teil G: Dr. Eugen Klever, Dr. Helene Fröhlich.

Teil $H$ : Dr. Gerd Bauer, Dr.-Ing. habll. Paul Eckert, Dr. Mechthilde Gehlen-Keller, Dipl.-Ing. Frigga Thomas, Dr. Michael Winiker. Auswärtiger wissenschaftlicher Mitarbeiter: Dr.-Ing. Rudolf Karl Müler.

Slawische Sprachen: Dr. Helene Fröhlich, Dr. Georg Froelich, Dr. Eugen Klever, Dipl.-Ing. Otto Lebtag, Prof. Dr. Max Ulmann, Dr.-Ing. Christian Weiske.
Fernöstliche Sprachen (Chinesisch, Japanisch u.a.): Dr. Walther Metzener. Auswärtige wissenschaftliche Mitarbeiter: Prof. Dr.-Ing. Eturô Maekawa, Prof. Dr. Leonhard Schuler.

Patente: Dr. Julius Gante. Auswårtige wissenschaftliche Mitarbeiter: Dr.-Ing. Hermann Bräucker, Dr. Hanns Donle, Dr. Walther Ganzlin, Dr..Ing. Helmut Högel, Dr.-Ing. Josef Mühleisen, Dr.-Ing. Hans Jürgen Nitzschke.

Register: Dr. Else Arnold und Dr. Walther Schicke unter Mitarbeit von Dr. Else Brandt, Dr. Helene Fröhlich, Dr. Margret Hänel, Dipl.-Chem. Ursula Krüger, Dr. Gerda von Krueger, Dr.-Ing. Ruth Levi, Dr.-Ing. Günter Poetzscher, Dipl.-Chem. Wolfgang Räck, Dipl.-Ing. Elfriede Rosdorff, Dr.-Ing. Wolfgang Schramm, Dr.-Ing. Klaus Ullmann, Dr. Maria Winter.

Wissenschaftliche Mitarbeiter: Dr. Christa Bahr, Dipl.-Ing. Inge Buchta, Dipl.-Chem. Slgrid Dehmlow, Dipl.-Ing. Ingeborg Geibler, Dipl.-Chem. Gertraut Herper, Dipl.-Ing. Werner Hoffmann, Dipl.-Ing. Eva Hunger, Gertrud Klesse, Dipl.-Ing. Steffi Mohrmann, Dipl.-Ing. Marletta Moldenschardt, Dr. Martln Negwer, Dipl.-Chem. Sigrid Oelsner, Dipl.-Chem. Rosemarie Pankow, Dipl.Chem. Asta Reisner, Dipl.-Ing. Erika Repp, Margret Rodegast, Dr. Ingeborg Störig, Dipl.-Phys. Herbert Weber, Dipl.-Chem. Irmela Wendlandt, Dipl.-Chem. Rosemarie Werner, Dr. Irma Wilke.

Teohnlsche Redaktion elnschl. Referenten betreuung: Alice Hawelek, Berlin N 4, Schiffbauerdamm 19

\section{Sprachenbezeichnung in den Referaten des Chemischen Zentralblattes}

(Abkürzungen laut DIN-Normblatt 1502 Beiblatt 1)

Albanisch $=$ Alb.
Bulgarisch $=$ Bulg.
Chinesisch $=$ Chin.
Dänisch $=$ Dān.
Deutsch $=$ Dt.
Englisch = Engl.
Estnisch = Estn.
Finnisch $=$ Finn.
Flämisch = Flām.
Französisch $=$ Franz.
Griechisch $=$ Griech.
Hollåndisoh = Holl.
Irisoh $=$ Ir.
Italienisch $=$ Ital.
Japanisch $=$ Jap.

Albanisch $=$ Alb.

Bulgarisch $=$ Bulg.

Chinesisch $=$ Chin.

Dänisch = Dān.

Englisch = Engl.

Estnisch = Estn.

Finnisch $=$ Finn.

Flämisch $=$ Fläm.

Griochish

Hollåndisoh $=$ Holl.

Irisch $=$ Ir.

Japanisoh $=$ Jap.

$\begin{array}{ll}\text { Lateinisch } & =\text { Lat. } \\ \text { Litauisch } & =\text { Litau. } \\ \text { Norwegisch } & =\text { Norw. } \\ \text { Persisch } & =\text { Pers. } \\ \text { Polnisch } & =\text { Pol. } \\ \text { Portugiesisch } & =\text { Port. } \\ \text { Rumänisch } & =\text { Rum. } \\ \text { Russisch } & =\text { Russ. } \\ \text { Schwedisch } & =\text { Schwed. } \\ \text { Serbokroatisoh } & =\text { Serbokroat. } \\ \text { Slowakisch } & =\text { Slowak. } \\ \text { Slowenisch } & =\text { Slowen. } \\ \text { Spanisch } & =\text { Span. } \\ \text { Tschechisch } & =\text { Tschech. } \\ \text { Türkisch } & =\text { Türk. } \\ \text { Ukrainisch } & =\text { Ukrain. } \\ \text { Ungarisch } & =\text { Ung. }\end{array}$




\section{CHEMISCHES ZENTRALBLATT}

VOLLSTÄNDIGES REPERTORIUM

FƯR ALLE ZWEIGE DER REINEN UND ANGEWANDTEN CHEMIE

1830 gegründet

1897-1945 von der Deutschen Chemischen Gesellschaft fortgeführt

In Auftrage der Deutschen $\mathbf{A k a d e m i e}$ der Wissensehaften zu Berlin, der Chemischen Gesellschait in der DDR, der Akademie der Wissensehaften zu Göttingen und der Gesellschaft Deutscher Chemiker (in der BRD) herausgegeben von

Prof. Dr. Heinrich Bertsch und Prof.Dr.Dr.h.c.Wilhelm Klemm

133. JAHRGANG

1962

SEITE $10337-11796$

Chefredakteure: Dr. Eugen Klever und Dr. WIIII Barz

1962

AKADEMIE-VERLAG GMBH - BERLIN VERLAG CHEMIE • GMBH・WEINHEIMIBERGSTR. 


\begin{tabular}{|c|c|c|c|}
\hline Heft-Nr. & Seitenzahl & \multicolumn{2}{|c|}{ Ausgabedatum } \\
\hline 29 & $10337-10716$ & 18. Juli & 1962 \\
\hline 30 & $10717-11072$ & 25. Juli & 1962 \\
\hline 31 & $11073-11436$ & 1. August & 1962 \\
\hline 32 & $11437-11796$ & 8. August & 1962 \\
\hline
\end{tabular}

Herausgeber: Prof. Dr. Helnrich Bertsch, Berlin, u. Prof. Dr. Dr.h.c. Wilhelm K lem m, Munster. Chefredakteure und verantwortlich fü den Inhalt: Dr. E u ge $\mathrm{K}$ lever, Berlin W 30 , Geisbergstr. 39 , Fernsprecher: 2495 41, und Dr. Willi Barz, Berlin N 4, Schiffbauerdamm 19, Fernsprecher: 425571.

(C)

Verlag: Akademie-Verlag GmbH, Berlin W 8, Lelpziger Str. 3-4 (Kernsprecher: 220441, Telex-Nr. 011773 , Postscheckkonto Berlln 35021), und Verlag Chemie, G mbH, Weinheim/Bergstr. (Fernsprecher: Weinheim 3635 [Sammelnummer]; Fernschrelber: 0465516 chemieverl wnh; Postscheckkonten: Frankfurt/M. 3635
145314
u. Berlin [West] 7430). Bestellnummer: 1007/133. Das Chemische Zentralblatt erscheint wóchentlich. Preis des Jahrganges einschl. Autoren-, Patent-, Sach- u. Formelreglster: DM 1200,-; einseltig bedruckte Ausgabe: DM 1350,- . Abgabe der Zeitschrift nur mit såmtlichen Registern.

Abbestellungen nur bís spätestens $\boldsymbol{B}$ Wochen vor Ablauf des Kalenderjahres.

Alle Rechte, auch die der tbersetzun sowio der photomechanischen Wiedergabe, sind vorbehalten. All rights reserved (including those of translations into foreign languages). No part of thls issue may be reproduced in any form, by photoprint, microfilm or any other means, witbout written permission Irom the publishers.

Die Wiedergabe von Gebrauchsnamen, Handelsnamen, Warenbezeichnungen usw. in diesen Heften berechtigt auch ohne besondere Kennzeichnung nleht zu der Annahme, daB solche Namen im Sinne der Warenzeichen- und Markenschutz-Gesetzgebung als freí zu betrachten wären und daher von jedermann benutzt werden dürten.

Satz und Druck: $\nabla$ EB Leipziger Druckhans, Leipzic. III/18/203, 7/62

Veroffentlicht unter der Lizenznummer ZLN 5002 des Ministeriums fir Kultur, Hauptverwaltung Verlagswesen. Printed in Germany.

Die Aufgabe von Anzeigen kann sowohl beim Akademie-Verlag, Berlin, als auch beim Verlag Chemie GmbH, Weinheim/Bergstr., erfolgen 